\title{
ADENOMA VELLOSO EN COLOCISTOPLASTIA DE AUMENTO ASOCIADO A TUMOR UROTELIAL INFILTRANTE EN EL REMANENTE VESICAL
}

Adrian Husillos Alonso, David Subirá Ríos, Roberto Molina Escudero y Carlos Hernández Fernández.

Servicio de Urología. Hospital General Universitario Gregorio Marañón. Madrid. España.

Resumen.- OBJETIVO: Presentación de un nuevo caso de adenoma velloso desarrollado en una colocistoplastia de aumento.

MÉTODO: Caracterización de un nuevo caso y revisión de la literatura publicada hasta la fecha.

RESULTADOS: Presentamos el caso de un varón de 66 años con un adenoma velloso sincrónico con un tumor infiltrante de urotelio vesical y que había sido sometido a una colocistoplastia de aumento por microvejiga tuberculosa. El tiempo de latencia desde la cirugía hasta su aparición fue de 23 años. El tratamiento consistió en su resección transuretral.

Adrián Husillos Alonso

Secretaría de Urología.

Hospital General Universitario

Gregorio Marañón

Dr. Esquerdo 46

28007 Madrid (España).

adrhusillos@hotmail.com

Aceptado para publicar: 12 de marzo 2010. 
CONCLUSIONES: El adenoma velloso es un tumor benigno de la mucosa colónica con alto potencial para evolucionar hacia un cáncer de colon infiltrante. Su aparición en colocistoplastias de aumento ha sido previamente descrita en la literatura en dos ocasiones. Recomendamos seguimiento estrecho mediante cistoscopia, dada su capacidad de malignización.

Palabras clave: Adenoma velloso. Colocistoplastia de aumento.

Summary.- OBJECTIVE: To report a new case of villous adenoma developed in augmentation colocystoplasty.

METHODS: Characterization of a new case and review of the literature published to date.

RESULTS: We report the case of a 66 year-old man with a villous adenoma and synchronic infiltrating transitional cell carcinoma of the bladder after augmentation colocystoplasty. The latency period until the development of villous adenoma after surgery is long. Treatment consisted of transurethral resection.

CONCLUSIONS: Villous adenoma is a benign neoplasm that occurs in the colonic mucosa and shows a high ability to become a malignant colonic cancer. Only two cases of villous adenoma in augmentation colocystoplasty have been reported. We recommend follow up with periodic cystoscopy because of its high malignancy potency.

Keywords: Villous adenoma. Augmentation colystoplasty.

\section{INTRODUCCIÓN}

El desarrollo de tumores en los segmentos intestinales empleados en cirugías de ampliación, derivación o sustitución vesical es una complicación tardía bien conocida de estos procedimientos (1). El primer caso fue descrito por Hammer en el año 1929 en una vesicosigmoidostomía tras 10 años de seguimiento (2).

Presentamos el caso de un adenoma velloso en una colocistoplastia de aumento que coexiste con un tumor infiltrante de vejiga. En nuestro conocimiento, es el tercer caso de adenoma velloso en una colocistoplastia de aumento publicado en la literatura hasta la fecha.

\section{CASO CLÍNICO}

Varón de 66 años con antecedentes de hipertensión arterial y diabetes mellitus tipo 2. Así mismo, es fumador de cigarrillos de cuarenta años de evolución.
Como antecedentes urológicos, había sido sometido a una nefroureterectomía derecha y colocistoplastia de aumento en el año 1986 por secuelas postuberculosis urinaria.

El paciente acude al servicio de urgencias de nuestro centro por un cuadro de dolor lumbar izquierdo y oligoanuria de una semana de evolución.

En las pruebas complementarias solicitadas se objetiva:

- Creatinina sérica 4,2; urea 111 ;

- Ecografía: dilatación moderada de la vía urinaria izquierda y engrosamiento parietal vesical en la zona de la desembocadura ureteral izquierda.

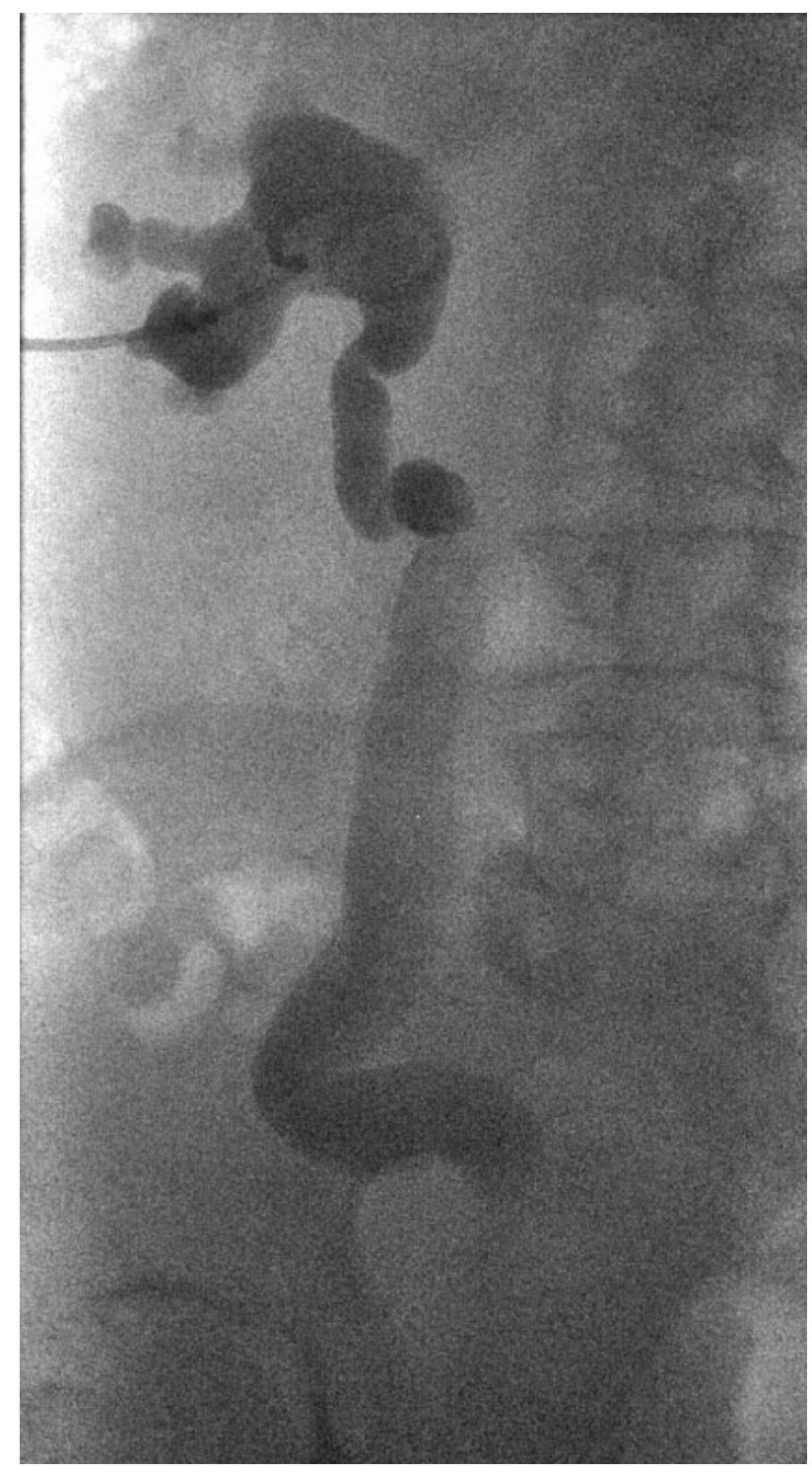

FIGURA 1. Imagen de pielografía anterógrada: vía urinaria dilatada. 
Con el diagnóstico de fallo renal agudo de causa obstructiva, se lleva a cabo la colocación de nefrostomía percutánea urgente, con posterior recuperación de la función renal previa.

Posteriormente, una vez recuperado del proceso agudo, se llevan a cabo los siguientes estudios:

- Pielogragfía anterógrada: se observa estenosis obstructiva en el uréter distal sin paso de contraste a vejiga (Figura 1).

-TAC-urografía (tomografía axial computerizada con fase de eliminación de contraste): se observa un engrosamiento de la pared vesical en la zona del meato ureteral izquierdo compatible con un tumor vesical.

Con el diagnóstico de probable tumor vesical en la región meática izquierda, se lleva a cabo exploración endoscópica bajo anestesia, con el hallazgo de una lesión sugestiva de un tumor vesical en el hemitrígono izquierdo de aspecto sólido e infiltrante y con afectación del meato izquierdo; así como una lesión pediculada dependiente de la mucosa de la colocistoplastia (Figura 2).

Se realizó una resección transuretral completa de ambas lesiones.

El resultado anatomopatológico de la lesión hemitrigonal fue un tumor de urotelio infiltrante T2G3. La toma correspondiente a la lesión polipoidea presentó una mucosa colónica con vellosidades de papilas individualizadas revestidas de elementos cilíndricos de citoplasma débilmente eosinófilo, con diagnóstico de adenoma velloso.

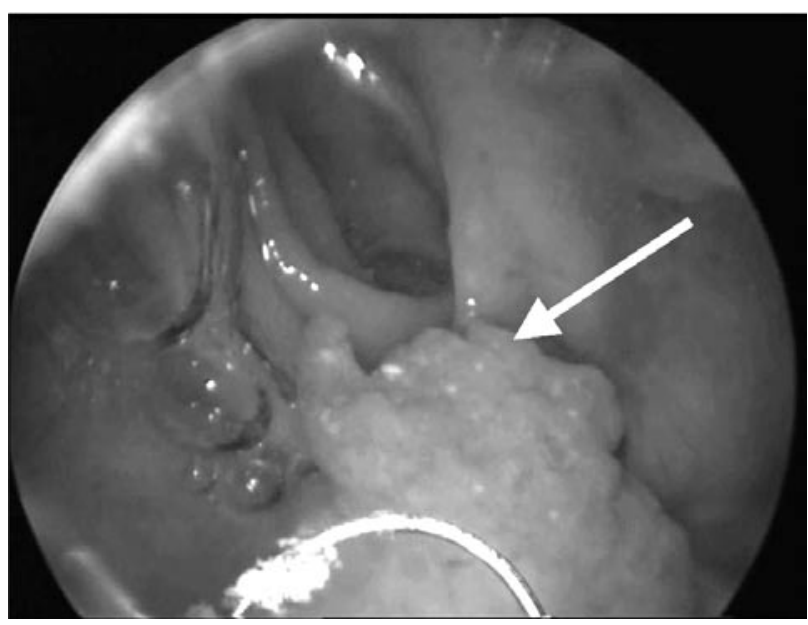

FIGURA 2. Imagen de adenoma velloso durante la resección transuretral de vejiga.
Se llevó a cabo cistoprostatectomía radical y derivación urinaria tipo ureterostomía cutánea; encontrándose libre de enfermedad hasta el momento actual.

\section{DISCUSIÓN}

Hasta la fecha han sido descritos quince casos de tumores en colocistoplastias: ocho adenocarcinomas, uno de ellos simultáneo con un carcinoma transicional, dos adenocarcinomas de células transicionales, dos adenocarcinomas de células en anillo de sello, un carcinoma microcítico y dos adenomas vellosos (1).

La mayoría de estos tumores aparecieron en la zona de la anastomosis del segmento de colon con la pared vesical (2). El tiempo medio de latencia desde la cirugía hasta el diagnóstico fue de 17 años en una revisión de todos los tumores desarrollados en colocistoplastias $(0,25-27)(1)$.

Se desconoce la etiopatogenia de estos tumores, aunque se sugieren noxas que actúan durante largos periodos, como la inflamación crónica en la zona anastomótica (3). También se ha relacionado la tuberculosis con la aparición de tumores en el tracto urinario $(4,5)$.

El adenoma velloso es un tumor benigno del colon que asienta más frecuentemente en la mucosa rectosigmoidea (6). Tiene un alto potencial de malignización, con un RR de 8,34 respecto de otros pólipos intestinales para el desarrollo de cáncer de colon durante el seguimiento (7).

Su aparición en colocistoplastias de aumento ha sido previamente documentada sólo en dos casos. Las características de los dos casos descritos se resumen en la Tabla I $(8,9)$.

En nuestro caso, encontramos un adenoma velloso dependiente de la zona cercana a la anastomosis tras 23 años de periodo de latencia, sincrónico con un tumor infiltrante de vejiga dependiente del remanente vesical. El tratamiento definitivo consistió en cistectomía radical y derivación tipo ureterostomía cutánea.

Dado que el tiempo de latencia hasta su aparición es largo, es probable que aparezcan nuevos casos conforme aumente el tiempo de seguimiento de los pacientes sometidos a cirugía de ampliación vesical. El adenoma velloso no es distinguible por técnicas de imagen de otros tumores, por lo que precisa exploración bajo anestesia y resección transuretral del mismo. El aspecto macroscópico es similar a un pólipo intestinal que depende de la mucosa colónica. Ya que en nuestro caso se da una coexistencia con un tumor infiltrante de vejiga, se ha llevado a cabo cistectomía radical, lo que ha imposibilitado el seguimiento y diagnóstico de una posible recidiva o progresión a cáncer de colon del adenoma velloso. 
TABAL I. CARACTERÍSTICAS DE ADENOMAS VELLOSOS PREVIAMENTE PUBLICADOS.

\begin{tabular}{|c|c|c|c|c|}
\hline Referencia & $\begin{array}{c}\text { Periodo de } \\
\text { latencia }\end{array}$ & Anatomía patológica & $\begin{array}{c}\text { Lugar de asiento } \\
\text { del tumor }\end{array}$ & Tratamiento \\
\hline Gepi-Atte et al. (8) & 25 años & Adenoma velloso & Anastomosis & Cistectomía parcial \\
\hline Yip et al. (9) & - & $\begin{array}{c}\text { Adenoma velloso } \\
\text { sincrónico con un } \\
\text { adenocarcinoma } \\
\text { mucinoso de pelvis renal }\end{array}$ & Anastomosis & $\begin{array}{c}\text { RTU para el adenoma } \\
\text { velloso y nefrourete- } \\
\text { rectomía para el tumor } \\
\text { de pelvis renal }\end{array}$ \\
\hline
\end{tabular}

\section{CONCLUSIÓN}

En resumen, el diagnóstico de un adenoma velloso en un segmento intestinal empleado como derivación urinaria obliga a un correcto tratamiento. La resección transuretral podría se un tratamiento adecuado; ya que permite la conservación de función mediante una técnica poco invasiva.

Puesto que el adenoma velloso es una lesión con elevado potencial de malignización, recomendamos su seguimiento a largo plazo mediante cistoscopia.

\section{BIBLIOGRAFÍA y LECTURAS RECOMENDADAS (*lectura de interés $y^{* *}$ lectura fundamental)}

**1. Austen M, Kälble T. Secondary malignancies in different forms of urinary diversion using isolated gut. J Urol, 2004; 172: 831-8.

2. Kälble T, Tricker AR, Friedl P, Waldherr R, Hoang A, Staehler G. et al. Ureterosigmoidostomy: longterm results, risk of carcinoma and etiological factors for carcinogenesis. J Urol, 1990; 144: 1110.
*3. Filmer RB, Spencer JR. Malignancies in bladder augmentations and intestinal conducts. J Urol, 1990; 143: 671-8.

4. Feeney D, Quesada ET, Sirbasku DM, Kadmon D. Transitional cell carcinoma in a tuberulous kidney: case report and review of the literature. J Urol, 1994; 151: 989.

5. Haddad FD. RE: Transitional cell carcinoma in a tuberulous kidney: case report and review of the literature (letter to the editor). J Urol, 1994; 152: 2105.

6. O’Brien MJ, Winawer SJ, Zauber AG, Gottlieb LS, Sternberg SS, Díaz B, et al. The National Polyp Study. Patient and polyp characteristics associated with high grade dysplasia in colorectal adenomas. Gastroenterology, 1990; 98: 371-9.

7. Yang G, Zheng W, Sun QR, Shu XO, Li WD, Yu H, et al. Pathologic features of initial adenomas as predictors for metachronous adenomas of the rectum. $\mathbf{J}$ Nati Cancer Inst, 1998; 90: 1661-5.

*8. Gepi-Attee S, Ganabathi K, Abrams PH, MacIver AG. Villous adenoma in augmentation colocystoplasty, a case report and discussion of the pathogenesis. J Urol, 1992; 147: 128.

*9. Yip SK, Wong MP, Cheung MC, Li JH. Mucinous adenocarcinoma of renal pelvis and villous adenoma of bladder after caecal augmentation of bladder. Aust NZJ Surg, 1999; 69: 247. 